\title{
On the relation between event-based and time-based current statistics
}

\author{
Massimiliano Esposito ${ }^{1,2}$, Katja Lindenberg $^{2}$ and Igor M. Sokolov ${ }^{3}$ \\ 1 Center for Nonlinear Phenomena and Complex Systems, Université Libre de Bruxelles, Code Postal 231, Campus \\ Plaine, B-1050 Brussels, Belgium. \\ 2 Department of Chemistry and Biochemistry and BioCircuits Institute, University of California, San Diego, La Jolla, \\ CA 92093-0340, USA. \\ 3 Institut für Physik, Humboldt-Universität zu Berlin, Newtonstrasse 15, D-12489 Berlin, Germany
}

PACS 05.70.Ln - Nonequilibrium and irreversible thermodynamics

PACS 05.40.-a - Fluctuation phenomena, random processes, noise, and Brownian motion

PACS 05.20.-y - Classical statistical mechanics

\begin{abstract}
Current statistics can be calculated in various ways. Event-based approaches use the statistics of the number of events occuring during a given time. Time-based approaches use the statistics of the time needed to reach a given number of events. By analyzing normal as well as anomalous statistics of nonequilibrium currents through a two level system in contact with two different reservoirs, we investigate the conditions under which these different statistics do or do not yield identical predictions. We rely on the continuous time random walk formulation introduced in our earlier work [Phys. Rev. E 77, 051119 (2008)].
\end{abstract}

Introduction. - The study of currents is of fundamental importance in statistical mechanics. Average energy and particle currents are central in determining whether a system is in equilibrium. Indeed, at equilib'rium the net probability current between any pair of system states is zero, i.e., detailed balance between all pairs of states is satisfied. This of course means that all net currents through the system are zero. External driving mechanisms bring a system out of equilibrium and may lead to non-zero currents. Examples of driving mechanisms include contacts with reservoirs of different temperatures or chemical potentials, and external forces. The resulting departure from detailed balance is responsible for an associated non-zero entropy production [1].

The study of current statistics of systems out of equilibrium has become an important tool to analyze small systems where fluctuations around average behavior can be significant. In macroscopic systems only average currents are of interest because fluctuations are too insignificant to be observable. However, in small systems the fluctuations of currents around the average provide rich information about the nonequilibrium dynamics of these systems, as evidenced by the considerable recent interest in the field of photon and electron counting statistics [2 6]. Furthermore, current fluctuations have recently been connected to the generalized version of the Second Law of thermodynamics via a variety of fluctuation theorems [7-12]. However, there is a fundamental aspect of current statistics that has, to our knowledge, not yet been fully explored, namely, the circumstances under which ergodic conditions are or are not satisfied. Ergodicity involves the equivalence between ensemble-averaged and time-averaged statistical properties at equilibrium. Here we extend the notion of ergodicity to nonequilibrium currents by exploring conditions under which event-based and time-based current statistics are asymptotically equivalent.

It is of particular interest to address these questions in the presence of anomalous statistics [13] that are known to significantly affect the ergodic properties of the system [14-17. Effects of anomalous statistics were first observed in averaged current measurements on macroscopic disordered semiconductors and amorphous solids, and were described in terms of continuous time random walks (CTRW) [18 20. Nowadays, anomalous statistics can be measured by photon counting experiments on single nanodevices such as blinking single quantum dots [21, single nanocrystals [14, and single molecules 22]. Since these experiments can give access to the full probability distribution of the fluctuations it becomes appropriate to extend the concept of ergodicity beyond the average to 
higher moments.

Definitions and main results. - To describe the issue, we introduce a system in which certain elementary events occur as time $t$ progresses, for instance, the net transfer of a mass or of a charge in or out of a system. The process is stochastic, that is, there is a distribution of the number of events occurring in a given time interval, or there is a distribution of times at which one observes a given number of events. The current associated with such events is usually defined as $I=k / t$ under the assumption that the number of events, $k$, scales linearly with time. We will call this the "normal" scaling, and generalize the definition of the current to recognize the possibility that the number of events does not grow linearly with time,

$$
I=\frac{k}{t^{\alpha}}
$$

Normal behavior thus corresponds to $\alpha=1$, and we call other scaling behaviors "anomalous". In particular, we focus on anomalous behavior with sublinear scaling, $0<\alpha<1$. As we shall see, this scaling is consistent with the CTRW formalism and, more specifically, with the subordination principle of so-called anomalous statistical processes 30 32].

The scaling information is not sufficient to describe the full statistics of the current. We need to be more precise about the nature of the distribution that defines the behavior of the current and current moments. The traditional approach to current statistics is to count the number of events that occur during a given time interval. Mathematically, this means that one treats $k$ as a random variable and $t$ as a parameter. The associated probability distribution is $\mathcal{P}_{t}(k)$, the probability that $k$ events occur in time $t$. The current moments according to this point of view are calculated as

$$
\left\langle I^{m}\right\rangle_{t} \equiv \frac{\left\langle k^{m}\right\rangle_{t}}{t^{\alpha m}}=\frac{1}{t^{\alpha m}} \sum_{k} k^{m} \mathcal{P}_{t}(k),
$$

where $m$ is a positive integer. The average current is given by the first moment, $\langle I\rangle_{t}$. The subscript serves as a reminder that time here is a parameter.

An alternative approach is to consider time to be the random variable and the number of events the parameter. The associated probability density is $\mathcal{P}_{k}(t)$, the probability that a time $t$ is needed to observe $k$ events. The current moments according to this point of view are calculated as

$$
\left\langle J^{m}\right\rangle_{k} \equiv{\frac{k^{m}}{\left\langle t^{\alpha m}\right\rangle_{k}}}_{k}=\frac{k^{m}}{\int d t t^{\alpha m} \mathcal{P}_{k}(t)} .
$$

With this approach, we use a different symbol for the current $(J)$ simply to stress the difference. The average current is now given by the first moment $\langle J\rangle_{k}$. We stress that (2) and (3) require different types of measurement of the current. The first measures the current during a fixed time interval $t$ and the second measures it until a given number of events $k$ has occurred. The statistics is of course obtained by repeating the experiment many times or by doing it simultaneously on independent copies of the system.

At this point it might be tempting to say that the current statistics are ergodic when asymptotically the statistics of events and the statistics of times lead to identical results,

$$
\lim _{t \rightarrow \infty} \frac{\left\langle k^{m}\right\rangle_{t}}{t^{\alpha m}}=\lim _{k \rightarrow \infty} \frac{k^{m}}{\left\langle t^{\alpha m}\right\rangle_{k}},
$$

that is,

$$
\lim _{t \rightarrow \infty}\left\langle I^{m}\right\rangle_{t}=\lim _{k \rightarrow \infty}\left\langle J^{m}\right\rangle_{k}
$$

To our knowledge this concept of ergodicity would be new for two reasons. First, it defines an equivalence of time and ensemble averages at the level of the currents instead of ordinary system observables. The time average of a current cannot be expressed in terms of the fraction of time that the system spends in a given state along a trajectory, as do conventional approaches to ergodicity (e.g., [15]). Furthermore, currents are not uniquely specified by the system states and require that the reservoirs responsible for the transitions be specified as well. Second, we have not restricted this statement to averages $(m=1)$ but have stated it for all moments $(m \geq 1)$.

One of our two main results of this paper is the following:

The ergodic condition (44) is satisfied for normal statistics, $\alpha=1$, but fails for anomalous statistics, $0<\alpha<1$.

Our second goal in this work is to introduce current statistics for which an ergodic condition is satisfied for the entire range $0<\alpha \leq 1$. This is achieved by dealing with the current even more directly, so that time as a random variable governed by the probability density $\mathcal{P}_{k}(t)$ enters through the inverse moments,

$$
\left\langle I^{m}\right\rangle_{k} \equiv k^{m}\left\langle t^{-\alpha m}\right\rangle=k^{m} \int d t \frac{\mathcal{P}_{k}(t)}{t^{\alpha m}} .
$$

An alternative ergodic condition would then be the assertion that asymptotically the statistics of events and the statistics of inverse times lead to identical results,

$$
\lim _{t \rightarrow \infty} \frac{\left\langle k^{m}\right\rangle_{t}}{t^{\alpha m}}=\lim _{k \rightarrow \infty} k^{m}\left\langle t^{-\alpha m}\right\rangle_{k}
$$

that is,

$$
\lim _{t \rightarrow \infty}\left\langle I^{m}\right\rangle_{t}=\lim _{k \rightarrow \infty}\left\langle I^{m}\right\rangle_{k}
$$

The second main result of this paper is the following:

The ergodic condition (7) is satisfied for all values $0<\alpha \leq 1$.

While we could describe and justify these assertions in a very general way for currents that scale as (11), it is 
more instructive to do so using a simple model that highlights the principal issues. Our model system is sketched in Fig. 1, and falls within the framework considered in Ref. [23].

The model. - The system consists of two levels, $i=0$ and $i=1$, connected to two "particle" reservoirs that we call left (L) and right (R). The nature of the "particles" does not matter other than that the current is associated with the flow of these particles between $\mathrm{L}$ and $\mathrm{R}$ through the two-level system. The nature of $\mathrm{L}$ and of $\mathrm{R}$ also does not matter other than that $\mathrm{L}$ and $\mathrm{R}$ must of course be different in some way. It is the asymmetry between the two that generates the nonequilibrium constraint. We have chosen the simplest possible system for our example, namely, one that allows the presence of either no particles or one particle but no more than one particle. Such a model can be viewed as a single-site asymmetric simple exclusion process 24 and has been used, for example, to model electron transport in a single-level quantum dot 25.

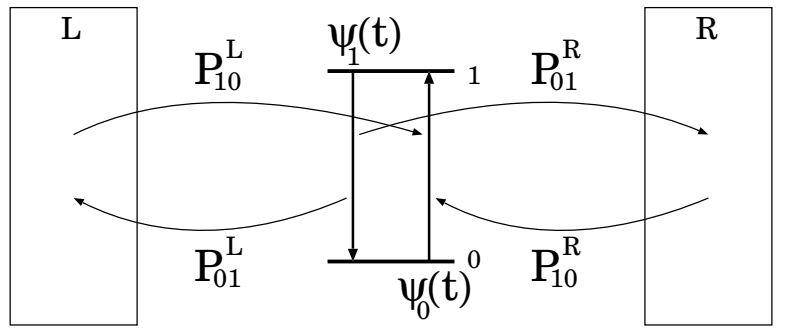

Fig. 1: Two-level system embedded between a left (L) and a right $(\mathrm{R})$ particle reservoir.

Transitions between the states of the system occur because of particle flow in or out of the system to one or the other reservoir. We follow the CTRW formulation in [23] (also called a semi-Markov process, e.g., [26, 27]) to describe these transitions. The transitions between the states are triggered by one or the other of the reservoirs, and since these are distinct and correspond to different mechanisms, we label the transitions with an index $\nu=$ $\mathrm{L}, \mathrm{R}$. Suppose that the system arrives at state $i^{\prime}$ at a given time and that its next transition is to state $i$ at a time $t$ later via mechanism $\nu$. We assume the distribution $\psi_{i i^{\prime}}^{\nu}(t)$ for this transition to be separable,

$$
\psi_{i i^{\prime}}^{\nu}(t)=P_{i i^{\prime}}^{\nu} \psi_{i^{\prime}}(t) .
$$

Here $P_{01}^{\nu}$ and $P_{10}^{\nu}$ are the transition probabilities downward and upward, respectively, triggered by reservoir $\nu$, and the waiting time distributions $\psi_{0}(t)$ for transitions from $i=0$ to $i=1$ and $\psi_{1}(t)$ for transitions from $i=1$ to $i=0$ are assumed to be independent of $\nu$.

To explore the consequences of anomalous statistics on various notions of ergodicity, we choose waiting time distributions that decay asymptotically as

$$
\psi_{i}(t) \sim\left(1 / \tau_{i}\right)\left(t / \tau_{i}\right)^{-\alpha-1} .
$$

The times $\tau_{0}$ and $\tau_{1}$ are characteristic times (but not in general first moments, which in fact diverge when $\alpha<1$ ). As we shall see shortly, this scaling is consistent with the scaling (11) of the current. The case $\alpha=1$ is representative of waiting time distributions that decay at least as fast as $1 / t^{2}$, and these have a first moment $\tau_{i}$. In Laplace space, to lowest order in $s$ the distributions behave as

$$
\tilde{\psi}_{i}(s)=1-\left(\tau_{i} s\right)^{\alpha} .
$$

We note that a direct connection to thermodynamics can be made for $\alpha=1$ by, for instance, identifying the two reservoirs as heat baths of equal temperatures $T$ but different chemical potentials $\mu_{\nu}$, and imposing the conditions $\left(P_{10}^{\nu} \tau_{1}\right) /\left(P_{01}^{\nu} \tau_{0}\right)=\exp \left(\mu_{\nu} / k_{B} T\right)$ [23]. A detailed discussion surrounding our choice of waiting time distribution can be found in 33 .

Current statistics. - Having described our setup, we are now ready to calculate the various statistical quantities needed to prove our statements about ergodicity. First, consider event-based statistics. We define the number of events $k_{\nu}$ to be the difference between the number of $0 \rightarrow 1$ and $1 \rightarrow 0$ transitions (denoted $N_{01}$ and $N_{10}$ ) triggered by the $\nu$ reservoir by time $t$, i.e.,

$$
k_{\nu}=N_{01} P_{01}^{\nu}-N_{10} P_{10}^{\nu} .
$$

It is always understood that a transition occurred at time $t=0$. In Ref. 23. we used a generating function formalism to obtain an analytic expression for the asymptotic form of the moments of $\mathcal{P}_{k_{\nu}}(t)$. Those results immediately lead to the result for the left hand sides of Eq. (4) [or (5D)] and Eq. (7) [or (8)],

$$
\lim _{t \rightarrow \infty}\left\langle I_{\nu}^{m}\right\rangle_{t}=\lim _{t \rightarrow \infty} \frac{\left\langle k_{\nu}^{m}\right\rangle_{t}}{t^{\alpha m}}=\frac{\Gamma(m+1)}{\Gamma(\alpha m+1)} a_{\nu}^{m},
$$

where

$$
a_{\nu} \equiv \frac{P_{10}^{\nu}-P_{01}^{\nu}}{\tau_{0}^{\alpha}+\tau_{1}^{\alpha}} .
$$

To calculate the right hand sides, we next consider the time-based statistics, that is, the statistics of the times needed to reach a given number of events. The probability $P_{k_{\nu}}(t)$ is the probability that $k_{\nu}$ events have occurred by time $t$ and is simply a time convolution of $N_{10}$ factors $\psi_{0}(t)$ and $N_{01}$ factors $\psi_{1}(t)$ in some order. The order is intertwined with the events triggered by the other reservoir, but does not matter when we go to Laplace space. Recalling that convolutions in the time domain lead to products in Laplace space, we get for the Laplace transform of $P_{k_{\nu}}$ the product

$$
\tilde{P}_{k_{\nu}}(s)=\left[\tilde{\psi}_{0}(s)\right]^{N_{10}}\left[\tilde{\psi}_{1}(s)\right]^{N_{01}} .
$$

The numbers of events $N_{10}$ and $N_{01}$ are random variables. However, this can be simplified at long times because whereas the total number of transitions $2 N=N_{01}+N_{10}$ 
is large, the difference is small, $\left|N_{01}-N_{10}\right| \leq 1$, the constraint coming from the fact that no more than one particle is allowed in the system. Therefore at long times we can set $N_{01} \approx N_{10} \approx N$, so that

$$
\tilde{P}_{k_{\nu}}(s)=\left[\tilde{\psi}_{0}(s) \tilde{\psi}_{1}(s)\right]^{N} .
$$

In the long time (small $s$ ) limit, Eq. (11) then leads to

$$
\tilde{P}_{k_{\nu}}(s)=\left[1-\left(\tau_{0}^{\alpha}+\tau_{1}^{\alpha}\right) s^{\alpha}\right]^{N},
$$

which can be approximated by

$$
\begin{aligned}
\tilde{P}_{k_{\nu}}(s) & \approx \exp \left[N \ln \left(1-\left(\tau_{0}^{\alpha}+\tau_{1}^{\alpha}\right) s^{\alpha}\right)\right] \\
& \approx \exp \left[-N\left(\tau_{0}^{\alpha}+\tau_{1}^{\alpha}\right) s^{\alpha}\right] \\
& =\exp \left(-\frac{k_{\nu}}{a_{\nu}} s^{\alpha}\right)
\end{aligned}
$$

In the last step we have set $k_{\nu} \approx N\left(P_{01}^{\nu}-P_{10}^{\nu}\right)$ and have used Eq. (14).

Equation (18) is the inverse Laplace transform of the one-sided Lévy distribution,

$$
\mathcal{P}_{k_{\nu}}(t)=\left(\frac{a_{\nu}}{k_{\nu}}\right)^{1 / \alpha} \mathcal{L}_{\alpha}\left[\left(\frac{a_{\nu}}{k_{\nu}}\right)^{1 / \alpha} t\right]
$$

The fractional moments of the Lévy distribution for $0<$ $\alpha \leq 1$ and $q<\alpha$ are [28,29]

$$
\int d u u^{q} \mathcal{L}_{\alpha}(u)=\frac{\Gamma(1-q / \alpha)}{\Gamma(1-q)},
$$

from which it follows that

$$
\left\langle t^{q}\right\rangle_{k_{\nu}}=\frac{\Gamma(1-q / \alpha)}{\Gamma(1-q)}\left(\frac{k_{\nu}}{a_{\nu}}\right)^{q / \alpha} .
$$

The higher positive moments $(q \geq \alpha)$ are singular. These moments allow us to calculate the right hand side of of Eq. (4) [or Eq. (5)]. In particular, the first immediate conclusion is that for $0<\alpha<1$ the moments in the denominator of the right hand side of Eq. (4) diverge. This means that the right hand side of this presumptive ergodicity condition vanishes, whereas the left hand side does not, cf. Eq. (13). Thus, the condition (4) [or (5)] indeed fails for anomalous statistics.

On the other hand, when $\alpha=1$ one finds that for any value of $q$

$$
\left\langle t^{q}\right\rangle_{k_{\nu}}=\left(\frac{k_{\nu}}{a_{\nu}}\right)^{q},
$$

and hence we find for the right hand side of Eq. (4) that

$$
\lim _{k_{\nu} \rightarrow \infty} \frac{k_{\nu}^{m}}{\left\langle t^{m}\right\rangle_{k_{\nu}}}=a_{\nu}^{m}
$$

This is identical to the result obtained from Eq. (13) when $\alpha=1$. Thus, this condition is satisfied for normal statistics.
Finally, we turn to the right hand side of Eq. (7) or (8). The negative moments can be obtained from Eqs. (20) and (21) with $q<0$. We immediately get

$$
\left\langle t^{-\alpha m}\right\rangle_{k_{\nu}}=\frac{\Gamma(m+1)}{\Gamma(\alpha m+1)} \frac{a_{\nu}^{m}}{k_{\nu}^{m}} .
$$

Using this result, we find that the time-based current statistics leads to the moments

$$
\lim _{k_{\nu} \rightarrow \infty}\left\langle I_{\nu}^{m}\right\rangle_{k_{\nu}}=\lim _{k_{\nu} \rightarrow \infty} k_{\nu}^{m}\left\langle t^{-\alpha m}\right\rangle_{k_{\nu}}=\frac{\Gamma(m+1)}{\Gamma(\alpha m+1)} a_{\nu}^{m} .
$$

The last expression is identical to that occurring in Eq. (13). This then proves the ergodic condition (7) or (8) for all $0<\alpha \leq 1$.

Conclusions. - In this letter we have considered the current statistics of systems away from equilibrium in which an external constraint produces a current. The waiting time distribution between successive elementary processes that give rise to the current decay as $t^{-\alpha-1}$ at long times, with $0<\alpha \leq 1$. The scaling is "normal" when $\alpha=1$ and "anomalous" when $0<\alpha<1$. The elementary events may, for example, be a transfer of particles or of charge. Specifically, we have addressed the question of the equivalence between different ways of calculating the current statistics.

Two setups can be used to directly measure a (properly scaled) fluctuating current $I=k / t^{\alpha}$. One can fix the time interval $t$ and measure the number $k$ of events occuring during this time or, alternatively, one can fix the number of events $k$ and measure the time $t$ required for this number of events to occur for the first time. We have shown that in both of these setups, the average current as well as the higher moments of the current are asymptotically the same, that is, $\lim _{t \rightarrow \infty}\left\langle k^{m}\right\rangle / t^{\alpha m}=\lim _{k \rightarrow \infty} k_{m}\left\langle t^{-\alpha m}\right\rangle$ for all $0<\alpha \leq 1$, cf. Eqs. (21), (6), and (8). A similar conclusion, but for a different model involving average particle velocities in tilted periodic potentials, has been reached in [34. When the current moments are calculated indirectly using the average time statistics $k^{m} /\left\langle t^{\alpha m}\right\rangle$, cf. Eq. (3), difficulties arise in the case of anomalous scaling. This occurs because this approach is in fact an incorrect way of calculating current moments except for normal scaling, where all three calculations of the current moments yield the same asymptotic results.

$$
* * *
$$

M. E. is supported by the FNRS Belgium (chargé de recherches) and by the government of Luxembourg (Bourse de formation recherches). I. S. thanks the DFG for support through the joint collaborative program SFB 555. K. L. gratefully acknowledge the support of the US National Science Foundation through Grant No. PHY0855471. 


\section{REFERENCES}

[1] J. Schnakenberg, Rev. Mod. Phys. 48, 571 (1976).

[2] C. W. Gardiner and P. Zoller, Quantum noise, 2nd ed. (Spinger, Berlin, 2000).

[3] E. Barkai, Y. Jung, and R. Silbey, Annu. Rev. Phys. Chem. 55, 457 (2004).

[4] F. Kulzer and M. Orrit, Annu. Rev. Phys. Chem. 55, 585 (2004).

[5] Yu.V. Nazarov, Ann. Phys. (Leipzig) 16, 720 (2007).

[6] M. Esposito, U. Harbola and S. Mukamel, Rev. Mod. Phys. 81, 1665 (2009).

[7] J. Kurchan, J. Phys. A 31, 3719 (1998).

[8] J. L. Lebowitz and H. Spohn, J. Stat. Phys. 95, 333 (1999).

[9] G. E. Crooks, Phys. Rev. E 60, 2721 (1999); Phys. Rev. E 61, 2361 (2000).

[10] U. Seifert, Phys. Rev. Lett. 95, 040602 (2005).

[11] D. Andrieux and P. Gaspard, J. Stat. Phys. 127, 107 (2007).

[12] M. Esposito, U. Harbola and S. Mukamel, Phys. Rev. E 76, 031132 (2007).

[13] R. Metzler and J. Klafter Phys. Rep. 339, 1 (2001).

[14] X. Brokmann, J.-P. Hermier, G. Messin, P. Desbiolles, J.P. Bouchaud, and M. Dahan, Phys. Rev. Lett. 90, 120601 (2003).

[15] G. Bel and E. Barkai, Phys. Rev. Lett. 94, 240602 (2005).

[16] A. Lubelski, I. M. Sokolov and J. Klafter, Phys. Rev. Lett. 100, 250602 (2008).

[17] Y. He, S. Burov, R. Metzler and E. Barkai, Phys. Rev. Lett. 101, 058101 (2008).

[18] H. Scher and M. Lax, Phys. Rev. B 7, 4491 - 4502 (1973); Phys. Rev. B 7, 4502 - 4519 (1973).

[19] H. Scher and E. W. Montroll, Phys. Rev. B 12, 2455 (1975).

[20] H. Scher, M. F. Shlesinger and J. T. Bendler, Physics Today 4426 (1991).

[21] M. Kuno, D. P. Fromm, S. T. Johnson, A. Gallagher, and D. J. Nesbitt, Phys. Rev. B 67, 125304 (2003).

[22] O. Flomenbom, K. Velonia, D. Loos, S. Masuo, M. Cotlet, Y. Engelborghs, J. Hofkens, A. E. Rowan, R. J. M. Nolte, M. Van der Auweraer, F. C. de Schryver, and J. Klafter, PNAS 102, 2368 (2005).

[23] M. Esposito and K. Lindenberg Phys. Rev. E 77, 051119 (2008).

[24] B. Derrida, Phys. Rep. 301, 65 (1998).

[25] M. Esposito, K. Lindenberg and C. Van den Broeck, EPL 85, 60010 (2009).

[26] C. Maes, K. Netočný and B. Wynants, J. Phys. A: Math. Theor. 42, 365002 (2009).

[27] D. Andrieux and P. Gaspard (2008), J. Stat. Mech. P11007.

[28] Gy. Terdik, W. A. Woyczynski, and A. Piryatinska, Phys. Lett. A 348, 94 (2006).

[29] F. Bardou, J.-P. Bouchaud, A. Aspect, and C. CohenTannoudji, Lévy Statistics and Laser Cooling (Cambridge University Press, 2003).

[30] M. M. Meerschaert, D. A. Benson, H.-P. Scheffler and B. Baeumer, Phys. Rev. E 65, 041103 (2002).

[31] A. A. Stanislavsky, Physica A 318, 469 (2003).

[32] R. Gorenflo, F. Mainardi and A. Vivoli, Chaos, Sol. Frac. 34, 89 (2007).
[33] M. F. Shlesinger, J. Stat. Phys., 10, 5 (1974).

[34] I. M. Sokolov, E. Heinsalu, P. Hänggi, and I. Goychuk, Europhys. Lett. 86, 30009 (2009). 